\title{
ADICIÓN DE LA PULPA DE CAFÉ EN LA DIETA DE OVINOS Y SU EFECTO EN LAS CARACTERÍSTICAS SEMINALES
}

\section{ADDING COFFEE PULP TO SHEEP'S DIET AND ITS EFFECT ON THE SEMINAL CHARACTERISTICS}

Recibido: 24 de septiembre 2021

Aceptado: 6 de noviembre 2021
R. Nieto Aquino ${ }^{1}$

P. Saldaña Campos ${ }^{2}$

B. Hernández Rodríguez ${ }^{3}$

R. Jiménez San Juan ${ }^{4}$

\section{RESUMEN}

El objetivo de este trabajo fue evaluar el efecto de la pulpa de café (PDC) en las características macro y microscópicas de semen ovino fresco y congelado colectado mediante vagina artificial. Diez carneros, fueron distribuidos aleatoriamente en dos tratamientos. El primero grupo SPC, $(n=5)$, con una dieta de mantenimiento, y el segundo grupo APC $(n=5)$ con un $5 \%$ de pulpa de café en la dieta. La recolección de semen se realizó utilizando una vagina artificial (VA), dos veces por semana en un horario de 7:00 am 8:00 am seguido del proceso de congelación. En los resultados no se encontraron diferencias significativas $(P<0.05)$ en todas las variables espermáticas evaluadas en semen fresco y congelado, volumen eyaculado, motilidad masal, motilidad individual, concentración espermática y prueba hipoosmótica. Se concluye que la inclusión de la pulpa de café al $5 \%$ en una dieta de mantenimiento para carneros no tiene efecto en las variables evaluadas en semen fresco y congelado.

PALABRAS CLAVE: Ovinos, reproducción, pulpa de café y características seminales.

\section{ABSTRACT}

The objective of this work was to evaluate the effect of coffee pulp (PDC) on the macro and microscopic characteristics of fresh and frozen sheep semen collected through an artificial vagina. Ten rams were randomly distributed in two treatments. The first group SPC, $(n=5)$, with a maintenance diet, and the second group APC $(n=5)$ with $5 \%$ of coffee pulp in the diet. Semen collection was performed using an artificial vagina (VA), twice a week from 7:00 am - 8:00 am followed by the freezing process. In the results, no significant differences were found $(\mathrm{P}<0.05)$ in all the sperm variables evaluated in fresh and frozen semen, ejaculate volume, mass motility, individual motility, sperm concentration and hyposmotic test. It is concluded that the inclusion of $5 \%$ coffee pulp in a maintenance diet for rams has no effect on the variables evaluated in fresh and frozen semen.

KEY WORDS: Sheep, reproduction, coffee pulp and seminal characteristics

\section{INTRODUCCIÓN}

La criopreservación del semen es una técnica que permite la conservación del material genético en los animales con el objetivo de ser utilizado en la inseminación artificial (IA); no obstante, la IA con semen congelado no presenta resultados favorables posiblemente al manejo durante la congelación y sus daños a nivel membrana espermática lo que ocasiona bajas en la fertilidad del rebaño (Barbas et al., 2013).

Las alteraciones son parcialmente producidas por el incremento en la producción de especies reactivas de oxígeno ROS durante el proceso de criopreservación, causando daños oxidativos en las biomoléculas que componen al espermatozoide, como peroxidación lipídica, oxidación

\footnotetext{
${ }^{1}$ Profesor del Tecnológico Nacional de México, Campus Huejutla Hidalgo, nietoaquinorafael@gmail.com.mx

${ }^{2}$ Profesor del Tecnológico Nacional de México, Campus Huejutla Hidalgo, panfilo.sc@ huejutla.tecnm.mx

${ }^{3}$ Profesor del Tecnológico Nacional de México, Campus Huejutla Hidalgo, blas.hr@ @huejutla.tecnm.mx

${ }^{4}$ Profesor del Tecnológico Nacional de México, Campus Huejutla Hidalgo, Roberto.js@ @huejutla.tecnm.mx
} 
de las proteínas y daños en el ADN. Sin embargo, se ha comprobado que en los procesos de conservación de semen, la adición de antioxidantes ha demostrado beneficios en las características del semen descongelado (Ruiz et al., 2007).

A la pulpa de café se le han atribuido algunas propiedades antioxidantes y es posible que algunas prácticas de manejo alteren su capacidad antioxidante (Arellano et al., 2011). Además de la cantidad de nutrientes presentes en la pulpa de café, el conocimiento del perfil de antioxidantes y de su capacidad antioxidante pudiera ayudar a disminuir o prevenir el daño oxidativo durante la crio preservación del semen, ya que la baja temperatura produce disminución de la actividad y concentración de enzimas antioxidantes (Yamauchi et al., 2013).

Por lo tanto, el objetivo del presente estudio fue evaluar el efecto de los antioxidantes presentes en la pulpa de café incluida en una dieta al 5\%, suministrada a sementales durante 2 meses durante el periodo de colección y evaluación de semen en fresco y en la postdescongelación en las características seminales de carneros.

\section{METODOLOGÍA}

\section{Descripción del área de estudio}

El presente experimento se desarrolló en la Unidad Ovina del Instituto Tecnológico de Huejutla, Hgo; localizado al norte del estado y geográficamente entre los paralelos $21^{\circ} 08^{\prime}$ de latitud norte y $98^{\circ} 25^{\prime}$ de longitud oeste, a una altitud de 140 metros sobre el nivel del mar, con un clima cálido - húmedo, una temperatura media anual de $31.1^{\circ} \mathrm{C}$ y la precipitación pluvial es de 1,500 milímetros por año.

\section{Animales y tratamientos}

El manejo de los animales se realizó de acuerdo a las normas de ética y bioseguridad del Consejo de Organizaciones Internacionales en Ciencias Médicas (CIOMS, 1986), en cumplimiento con la ley mexicana (NOM-062-ZOO-1999) para el uso de animales en experimentación (DOF, 2001).

Se utilizaron 10 sementales de la raza Dorset, de aproximadamente una edad de 1.5 años. Todos los carneros contaban con una condición corporal de 3 en una escala de 1 a 5, con un peso promedio de $80 \pm 5 \mathrm{~kg}$ y antecedentes de fertilidad probada, también se realizó manejo zootécnico el cual consistió en la esquila, despezuñado y corte de bellos prepuciales.

Los sementales tuvieron una etapa de entrenamiento de 4 semanas previas al establecimiento del experimento, periodo en el que se adaptaron a eyacular por las mañanas (7:00 - 8:00 AM), por medio de vagina artificial, en presencia humana y con ayuda de hembras estrogenizadas para facilitar la estimulación.

Posteriormente al periodo de capacitación de los sementales para la eyaculación, los carneros fueron distribuidos de manera aleatoria en dos grupos experimentales: 1) el grupo adicionado con pulpa de café ( $5 \%$, con base en materia seca) en la dieta (APC, n=5); 2) el grupo testigo sin la adición de pulpa de café en la dieta (SPC, $n=5)$. Ambas dietas se formularon para ser isoenergéticas e isoproteicas y cubrir los requerimientos de proteína cruda (9\%) y energía metabolizable (1.76 Mcal $\mathrm{kg}^{-1}$ ) de acuerdo a los requerimientos de mantenimiento en sementales ovinos (NRC, 2007). 
El periodo de alimentación de los sementales fue de 8 semanas, tomando la primera semana como adaptación a la dieta experimental. Los carneros fueron alojados en jaulas individuales de $1.2 \times 2.0 \mathrm{~m}\left(2.4 \mathrm{~m}^{2}\right)$, ofreciendo por animal $2.0 \mathrm{~kg} \mathrm{~d}^{-1} \mathrm{de}$ alimento $\left(1 \mathrm{~kg}\right.$ animal $^{-1} 08: 00$ am; $1 \mathrm{~kg}$ animal ${ }^{-1} 14: 00 \mathrm{pm}$, respectivamente), además de agua a libre acceso. La limpieza y las inspecciones de sanidad se realizaron periódicamente, durante el todo el periodo experimental.

\section{Recolección y Congelación del semen}

La recolección del semen se realizó una vez por semana a partir de que se comenzó a proporcionar la dieta a los animales, en un horario de 7:00 a 8:00 AM pasada la recolección el semen se registró el volumen eyaculado posteriormente se le adicionó el diluyente (1:200) a base de yema de huevo (20\%), triladyl (20\%) y agua destilada (60\%), se almacenó en los tubos de colección dentro de frascos completamente sellado a la luz para su posterior congelación.

Para la congelación, el semen se dejó un tiempo de 2 horas estabilizando a una temperatura de 5 grados aun dentro de los frascos cerrados en un refrigerador, pasado este tiempo se procedió al empajillado, que consistía en succionar el semen y después sellarlo para dejarlo estabilizar por otras 2 horas más a la misma temperatura, en total se obtuvieron 10 pajillas de $0.25 \mathrm{~mL}$ por cada semental dando un total 100 pajillas. Pasado este tiempo en una hielera de laboratorio se vertió nitrógeno hasta alcanzar una altura aproximada de 5 centímetros y se dejó reposar por 5 minutos hasta enfriarla completamente, posteriormente las pajillas se acomodaron en 2 gradillas con las mismas dimensiones y se metieron a la hielera para ser congeladas, la distancia entre el nitrógeno y las pajillas fue de tres centímetros por lo que fueron colocadas a una altura de 8 centímetros dentro de la caja, estas permanecieron 5 minutos con la caja abierta y 5 minutos cerrada después las pajillas se sumergieron completamente en el nitrógeno por 3 minutos para su posterior almacenamiento con la ayuda de globes y bastones en un termo especial.

La evaluación de la motilidad masal se realizó después de observar las muestras de semen diluido en el microscopio óptico a 40X, asignándole un valor subjetivo. Sin embargo, para determinar la motilidad individual fue necesario realizar una dilución previa (1:200) utilizando una solución salina formulada al 3\%, después de realizar la observación visual de las muestras a 40X, la motilidad individual fue calificada de manera subjetiva en la escala de 0 a $100 \%$, valorando el porcentaje de espermatozoides que presentan movimiento rectilíneo en un área determinada. La prueba de vivos y muertos se realizó con la ayuda de un frotis utilizando una gota de semen descongelado mezclado con eosina - nigrosina como colorante y facilitar la medición. Para medir la concentración espermática se utilizó un espectrofotómetro con una solución de $1 \mathrm{~mL}$ de semen fresco y $3 \mathrm{~mL}$ de agua destilada así evitando el error humano y obteniendo resultados más confiables.

\section{Análisis Estadístico}

El diseño experimental fue un DCA, donde cada semental fue considerado como unidad experimental, para las variables volumen por eyaculado, $\mathrm{pH}$, se realizó la prueba del PROC GLM y la prueba de comparación de medias de Tukey. Para la variable concentración espermática se realizó el procedimiento del PROC MIXED. Para la motilidad masal se realizó una prueba X2. Todos los procedimientos se realizaron con el paquete estadístico 
SAS versión 9 (SAS, 2009).

\section{RESULTADOS}

\section{Resultados de colección de semen}

\section{Volumen eyaculado}

Dados los resultados obtenidos en la variable volumen eyaculado, no se encontraron diferencias significativas estadísticas entre los promedios de los tratamientos experimentales (Con Pulpa $1.8 \mathrm{~mL}$; Sin Pulpa $1.7 \mathrm{~mL})(\mathrm{P} \geq 0.05)$, sin embargo, en el muestreo 3 y 4 es donde se pueden apreciar variabilidad entre los tratamientos (Figura 1). Los promedios obtenidos en este trabajo se encuentran dentro de los valores citados en un volumen eyaculado entre 0.8 a $2.0 \mathrm{~mL}$. Las variaciones que se muestran se podría deber a los factores medio ambientales, ya que la composición del semen varía considerablemente, entre especies y razas, incluso dentro de los mismos individuos según la época del año o las condiciones de explotación.

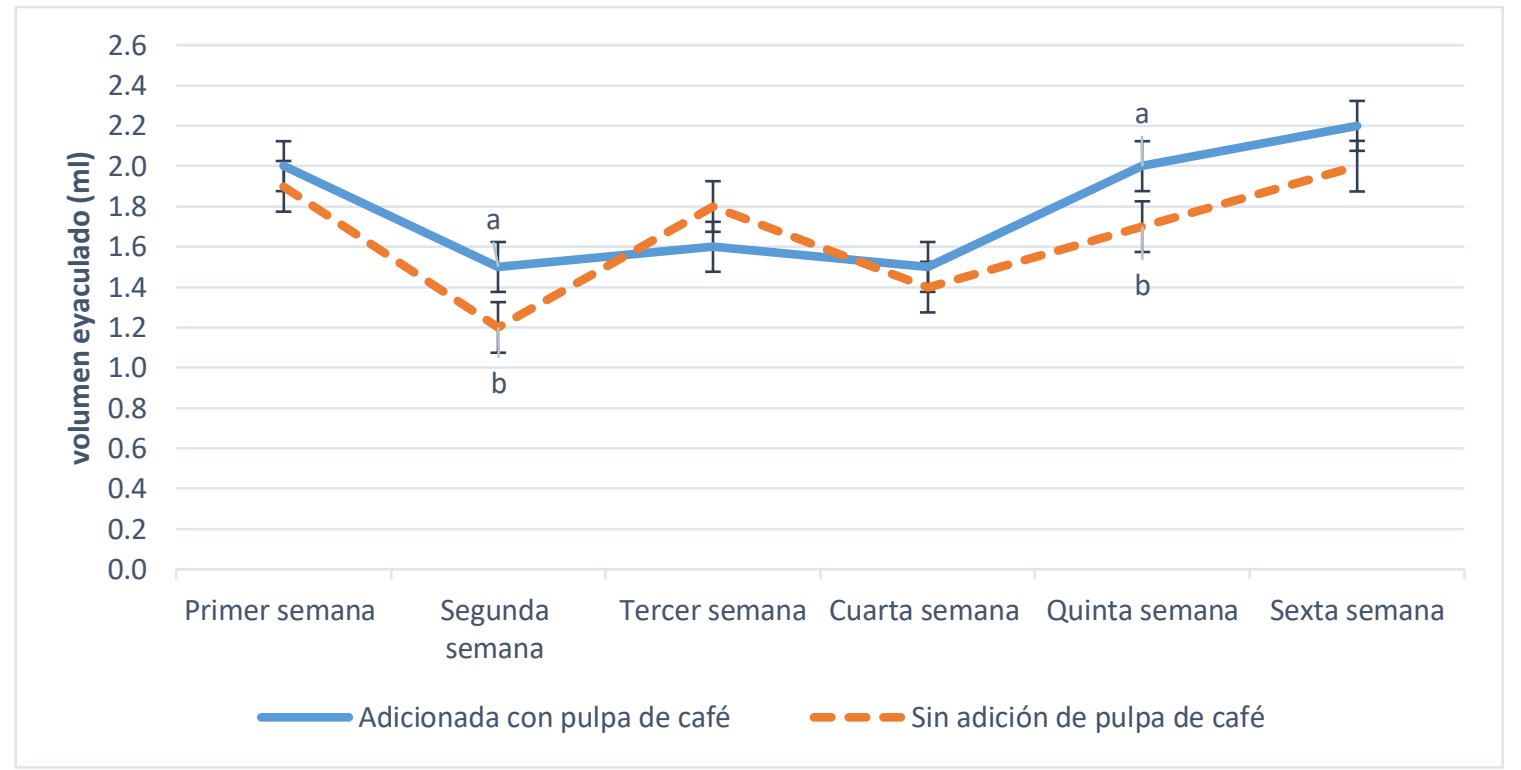

Figura 1. Promedios por semana de volumen eyaculado. T1: con pulpa de café, T2: sin pulpa de café, a y b son valores estadísticamente diferentes.

\section{Motilidad Masal}

Los promedios de la variable motilidad masal no presentaron diferencias significativas entre tratamientos experimentales (Con pulpa: 3.4; Sin pulpa: 3.3) ( $\mathrm{P} \geq 0.05)$, sin embargo, en los muestreos 3 y 4 se observan variantes estadísticas (Figura 2), lo que nos permite ver que la pulpa de café interviene de manera positiva en la motilidad masal del semen fresco bajo las condiciones adaptadas del experimento. 


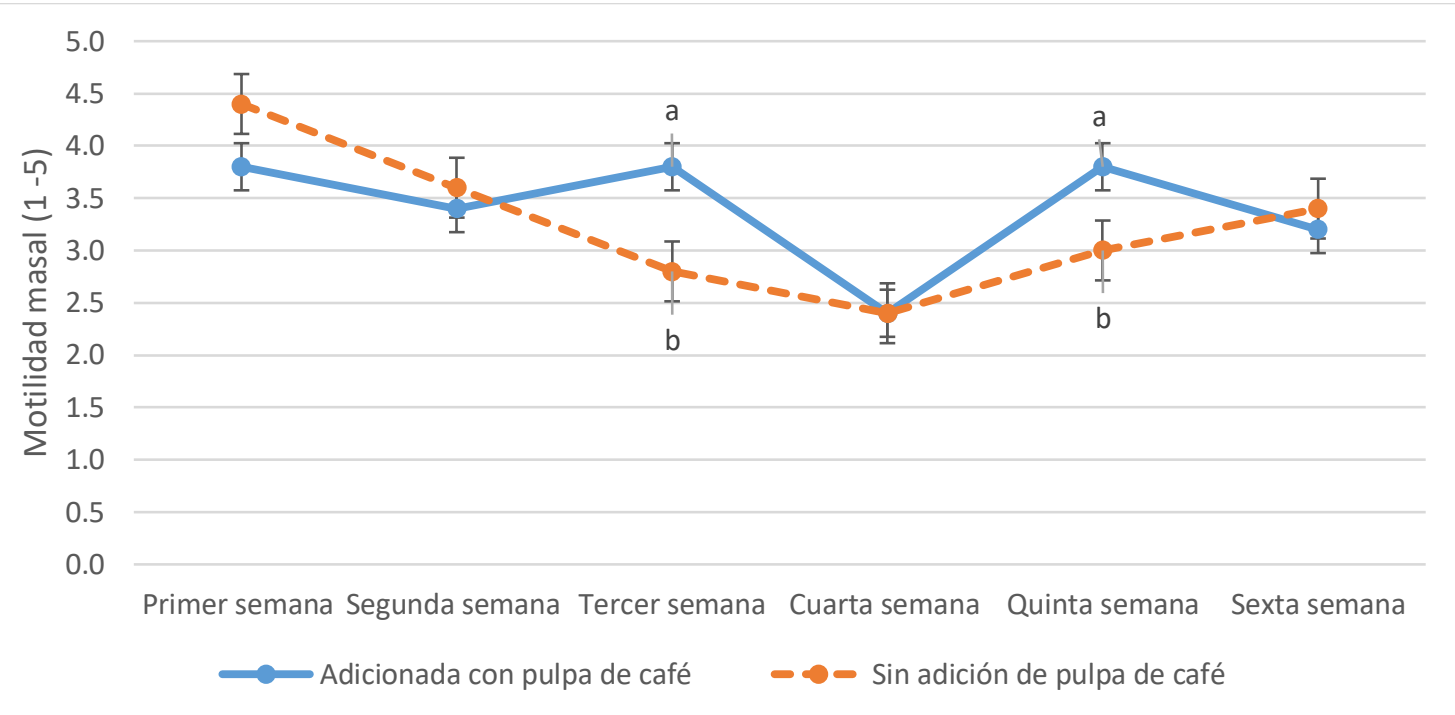

Figura 2. Promedios por semana de la Motilidad Masal. T1: con pulpa de café, T2: sin pulpa de café, a y b son valores estadísticamente diferentes

\section{Motilidad individual}

Los promedios obtenidos en la variable motilidad individual no mostraron diferencias significativas entre tratamientos (Con Pulpa: 66\%; Sin pulpa: 63\%) (P $\geq 0.05)$, sin embargo en el muestreo 3 mostro una diferencia estadística (Figura 3); cabe mencionar que no solamente resalta eso en el gráfico sino la súbita caída en la motilidad la semana cuarta lo cual puede deberse al incremento de la temperatura presente en la huasteca hidalguense.

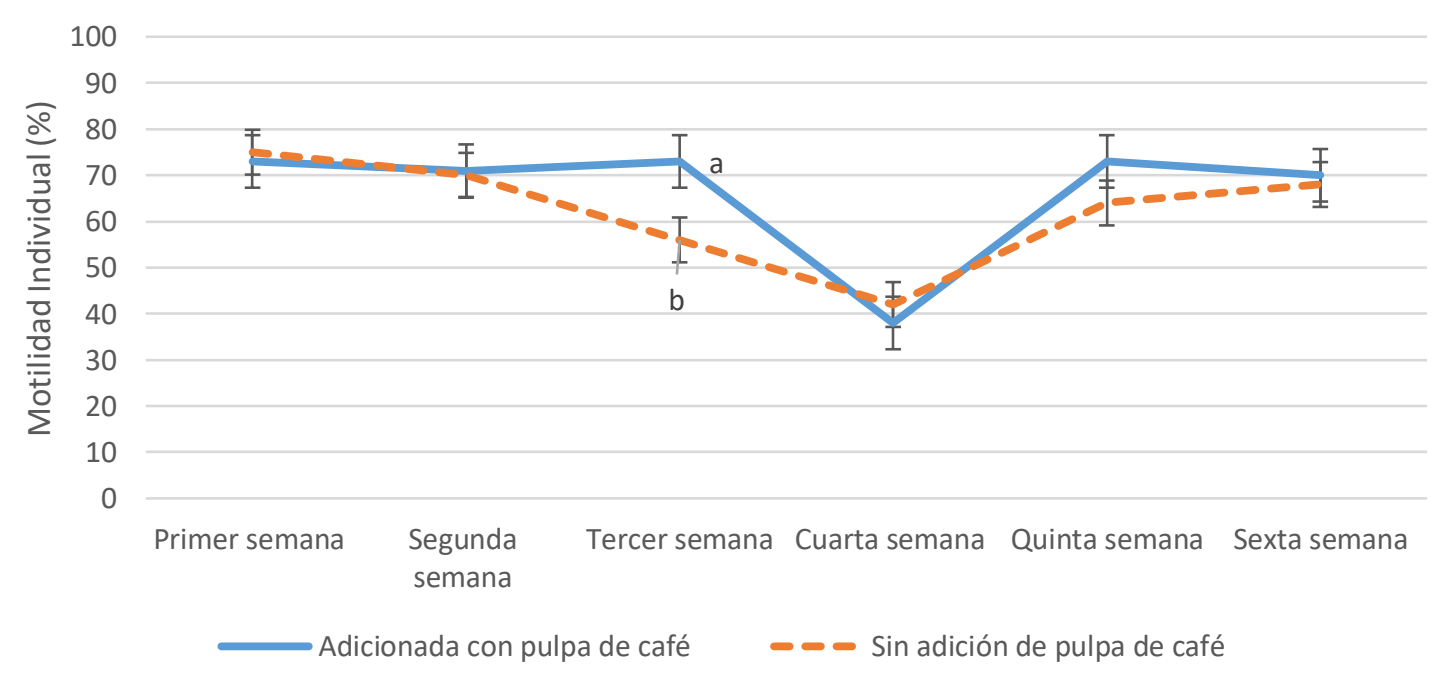

Figura 3. Promedios por semana de la Motilidad Individual. T1: con pulpa de café, T2: sin pulpa de café, a y b son valores estadísticamente diferentes

\section{Concentración espermática}

En la variable concentración espermática no se encontraron diferencias significativas entre tratamientos (Con Pulpa: 2.3 x109/ mL; Sin Pulpa $2.3 \times 109 / \mathrm{mL})(\mathrm{P} \geq 0.05)$ salvo en la quinta 
semana donde se acentúa una ligera diferencia estadística (Figura 4), aunque esto no determina la efectividad de la pulpa de café.

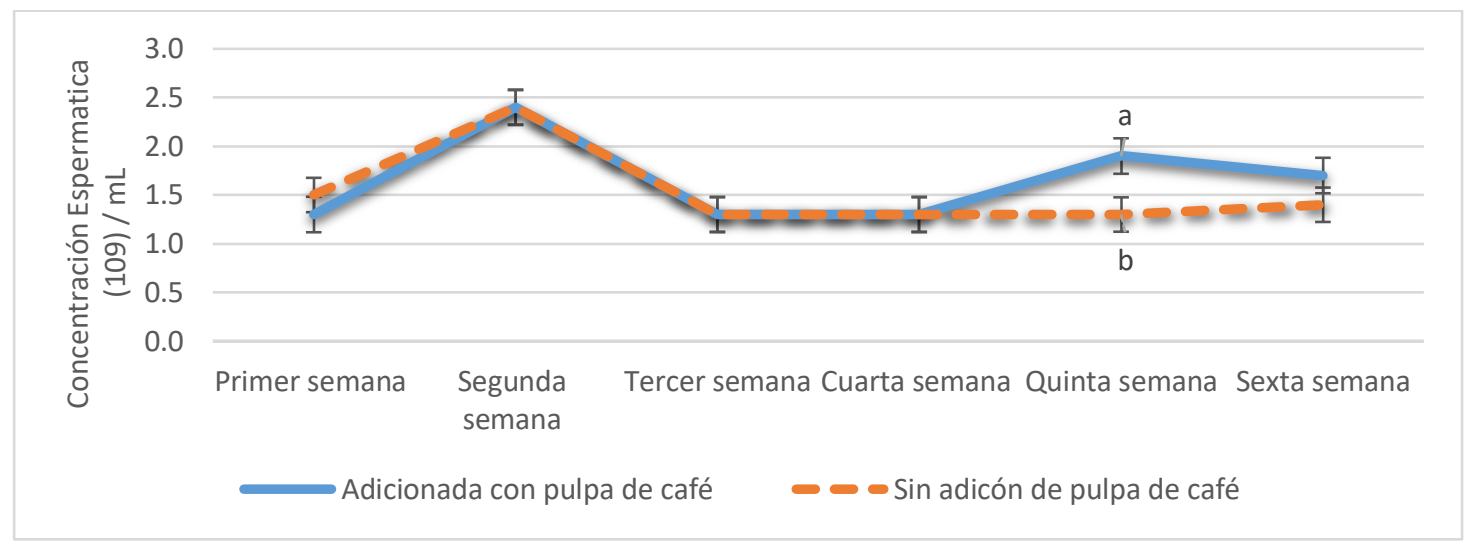

Figura 4. Promedios por semana de Concentración espermática. T1: con pulpa de café, T2: sin pulpa de café, a y b son valores estadísticamente diferentes

\section{Prueba Hipoosmótica después del congelado de pajillas}

Con respecto a los resultados obtenidos en esta variable no se encuentran diferencias significativas entre tratamientos (Con Pulpa: 77.8; Sin Pulpa: 78.6) $(\mathrm{P} \leq 0.05)$, sin embargo, se nota una diferencia en el tiempo enfatizando en la tercera semana (Figura 5); lo que nos permite afirmar que la pulpa de café no tiene un efecto en la Prueba Hipoosmótica a la descongelación del semen cuando se adiciona a una dieta de mantenimiento.

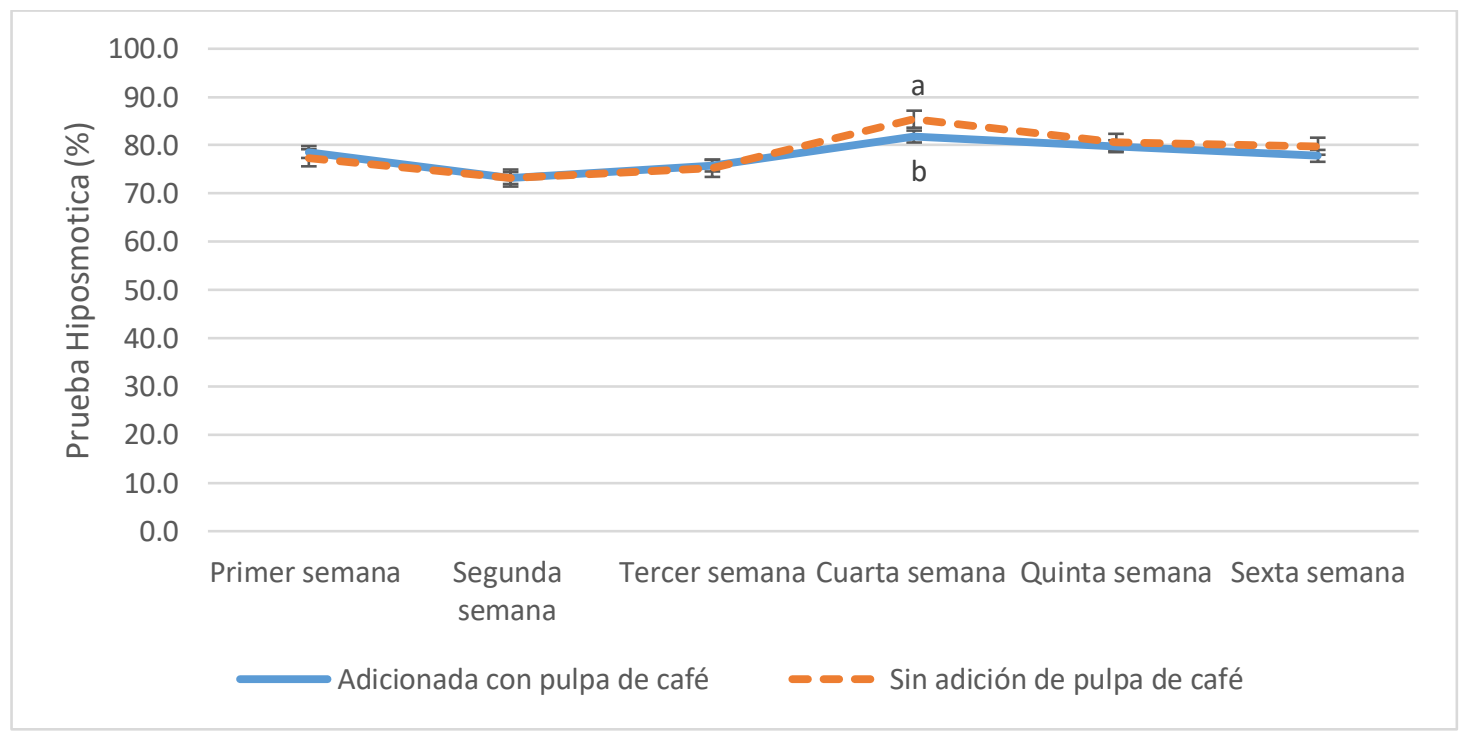

Figura 5. Promedios por semana de la Prueba Hipoosmótica de semen congelado. T1: con pulpa de café, T2: sin pulpa de café, a y b son valores estadísticamente diferentes 


\section{CONCLUSIONES}

Se concluye que la inclusión de la pulpa de café al 5\% en una dieta de mantenimiento para carneros no tiene efecto en las variables evaluadas en semen fresco y congelado. No obstante, se encuentra en la segunda etapa de investigación los análisis de la capacidad antioxidante que nos determinarán si se debe incrementar los niveles de pulpa de café en la dietas para nuevas investigaciones.

\section{AGRADECIMIENTOS}

Las deducciones de la presente investigación son resultados preliminares de un proyecto de investigación financiado por el Tecnológico Nacional de México en su convocatoria de Investigación y Desarrollo 2020.

\section{BIBLIOGRAFÍA}

Arellano G. M., Ramírez C.A., Torres M. T., Pérez M.G., Saucedo C.S.2011. Antioxidant Activity of Fermented and Nonfermented Coffee (Coffea arabica) Pulp Extracts. Food Technol. Biotechnol. 49 (3) 374-378.

Barbas J, Marques C, Baptista M, Mascarenhas R, Pereira R, CavacoGonçalves S. 2013. Fertilidade de carneiros de raça Saloia com sémen refrigerado ou congelado. Arch Zootec 62: 303-306. doi: 10.4321/S0004- 05922013000200018.

CIOMS (Council for international Organizations of Medical Sciences). 1986. "International Guiding Principles for Biomedical Research Involving Animals”. CIOMS, Geneva, Switzerland.

DOF (Diario Oficial de la Federación). 2001. "Norma Oficial Mexicana NOM-062-ZOO1999: Especificaciones técnicas para la producción, cuidado y uso de animales de laboratorio". México, D.F.

National Research Council (NRC). 2007. Nutrient requirements of small ruminants. Sheep, goats, cervids and new world camelids. National Academy Press, Washington D.C.

Ruiz L, Santiani A, Sandoval R, Huanca W, Delgado A, Coronado L, Alzamora C. 2007. Efecto de dos antioxidantes (tempo y tempol) en la criopreservación de semen ovino empleando un dilutor en base a Tris. Rev Inv Vet Perú 18: 99-106.

Statistical Analysis System (SAS). 2009. SAS/STATTM User's Guide, Release 5.0 Cary, N.C. U.S.A. SAS Inst. Inc.

Yamauchi, S., Suzuki, C., Noguchi, M., Kasa, S., Mori, M., Isozaki, Y., Ueda, S., 2012. Funahashi, H., Kikuchi, K., Nagai, T., Yoshioka, K. Effects of caffeine on sperm characteristics after thawing and inflammatory response in the uterus after artificial insemination with frozen-thawed boar semen. Theriogenology 79: 87-93. DOI: rights reserved. http://dx.doi.org/10.1016/j.theriogenology.2012.09.012. 